\title{
On the Computation of Some Topological Descriptors to Find Closed Formulas for Certain Chemical Graphs
}

\author{
Muhammad Haroon Aftab $\mathbb{D}^{1},{ }^{1}$ Muhammad Rafaqat $\mathbb{D}^{1},{ }^{1}$ M. Hussain $\mathbb{D},{ }^{2}$ and Tariq Zia $\mathbb{1 D}^{2}$ \\ ${ }^{1}$ Department of Mathematics, The University of Lahore, Lahore, Pakistan \\ ${ }^{2}$ Department of Mathematics, COMSATS University Islamabad, Lahore Campus, Islamabad, Pakistan
}

Correspondence should be addressed to Muhammad Haroon Aftab; haroon.aftab@math.uol.edu.pk

Received 20 February 2021; Accepted 2 May 2021; Published 21 May 2021

Academic Editor: Muhammad Imran

Copyright (C) 2021 Muhammad Haroon Aftab et al. This is an open access article distributed under the Creative Commons Attribution License, which permits unrestricted use, distribution, and reproduction in any medium, provided the original work is properly cited.

\begin{abstract}
In this research paper, we will compute the topological indices (degree based) such as the ordinary generalized geometricarithmetic (OGA) index, first and second Gourava indices, first and second hyper-Gourava indices, general Randic' index $R_{\gamma}(G)$, for $c=\{ \pm 1, \pm 1 / 2\}$, harmonic index, general version of the harmonic index, atom-bond connectivity $(\mathrm{ABC})$ index, $\mathrm{SK}, \mathrm{SK}{ }_{1}$, and $\mathrm{SK}_{2}$ indices, sum-connectivity index, general sum-connectivity index, and first general Zagreb and forgotten topological indices for various types of chemical networks such as the subdivided polythiophene network, subdivided hexagonal network, subdivided backbone DNA network, and subdivided honeycomb network. The discussion on the aforementioned networks will give us very remarkable results by using the aforementioned topological indices.
\end{abstract}

\section{Introduction}

The branch of mathematics that is related to the study of implementation of chemistry and graph theory together is called chemical graph theory. This theory is used to model the molecules of a chemical compound mathematically. This theory helps us to understand the physical properties of that chemical/molecular compound. In this theory, we construct the structure of a chemical compound in the form of a graph. In chemical graph theory, atoms are used as nodes, and bonds between the atoms are utilized as edges. A topological index is a numerical parameter of a graph that explains its topology. The topological index is also called a molecular descriptor and a connectivity index. It is obtained by transforming the chemical information into a numerical quantity. Topological indices are used as molecular descriptors in the construction of quantitative structure-activity relationships and quantitative structure-property relationships as well. The theoretical models such as quantitative structure-activity relationships (QSARs) relate the quantitative measure of a chemical structure to a biological property or a physical property, and quantitative structure-property relationships (QSPRs) relate mathematically physical/chemical properties to the structure of a molecule. Topological indices such as ordinary generalized geometric-arithmetic (OGA) index, first and second Gourava indices, first and second hyper-Gourava indices, general Randic index $R_{\gamma}(G)$, for $\gamma=\{ \pm 1, \pm 1 / 2\}$, harmonic index, general version of harmonic index $[1,2]$, atom-bond connectivity $(\mathrm{ABC})$ index $[3,4], \mathrm{SK}, \mathrm{SK}_{1}$, and $\mathrm{SK}_{2}$ indices, sumconnectivity index, general sum-connectivity index, and first general Zagreb [5] and forgotten topological indices have very significant roles in QSAR and QSPR studies and are used to discuss the bioactivity of molecular structures.

In 2009, D. Vukičević and B. Furtula established the first GA index in [6-11]. The first geometric-arithmetic (GA) index of a graph $\xi$ was calculated by

$$
\mathrm{GA}(\xi)=\sum_{g h \in E(\xi)} \frac{2 \sqrt{d_{g} d_{h}}}{d_{g}+d_{h}} .
$$

An ordinary geometric-arithmetic index of $\xi$ was produced in 2011 in [12] and formulated by, for each positive real number $k$, 


$$
\operatorname{OGA}_{k}(\xi)=\sum_{g h \in E(\xi)}\left[\frac{\sqrt{4 d_{g} d_{h}}}{d_{g}+d_{h}}\right]^{k}
$$

In 2017, V. R. Kulli proposed the first and second Gourava and hyper-Gourava indices in $[13,14]$. The first and second Gourava and hyper-Gourava indices of a graph $\xi$ were formulated by

$$
\begin{gathered}
\mathrm{GO}_{1}(\xi)=\sum_{g h \in E(\xi)}\left[\left(d_{g}+d_{h}\right)+\left(d_{g} d_{h}\right)\right], \\
\mathrm{GO}_{2}(\xi)=\sum_{g h \in E(\xi)}\left[\left(d_{g}+d_{h}\right)+\left(d_{g} d_{h}\right)\right], \\
\mathrm{HGO}_{1}(\xi)=\sum_{g h \in E(\xi)}\left[\left(d_{g}+d_{h}\right)+\left(d_{g} d_{h}\right)\right]^{2}, \\
\mathrm{HGO}_{2}(\xi)=\sum_{g h \in E(\xi)}\left[\left(d_{g}+d_{h}\right)+\left(d_{g} d_{h}\right)\right]^{2} .
\end{gathered}
$$

In 1975, Randic' index [15-17] was introduced by Milan Randic'. It is often used in chemoinformatics to investigate the compounds of chemicals. The Randic' index is also called "the connectivity index of the graph" and formulated by

$$
R_{(1 / 2)}(\xi)=\sum_{u v \in E(\xi)} \frac{1}{\sqrt{\left(d_{u} \cdot d_{v}\right)}}
$$

where $d_{u}$ and $d_{v}$ are the degrees of the nodes.

Later, Bollobás and Erdos furnished its generalized version for $\gamma$, where $\gamma \in R$, known as the general Randic' index [18-21] defined as

$$
R_{\gamma}(G)=\sum_{g h \in E(G)}\left(d_{g} \cdot d_{h}\right)^{\gamma}, \quad \text { for } \gamma=\left\{-1,1,-\frac{1}{2}, \frac{1}{2}\right\} .
$$

In 2012, L. Zhong described the harmonic index in $[22,23]$, and it is given by

$$
\mathrm{HI}(G)=\sum_{g h \in E(G)} \frac{2}{d_{g}+d_{h}} .
$$

In 2015, L. Yan introduced the general version of the harmonic index in [24] and defined by

$$
H_{k} I(G)=\sum_{g h \in E(G)}\left[\frac{2}{d_{g}+d_{h}}\right]^{k} \text {. }
$$

In 2008, Ernesto“ Estrada et al. [25, 26] introduced a new topological index, named atom-bond connectivity (ABC) index, calculated by

$$
A B C(G)=\sum_{g h \in E(G)} \sqrt{\frac{d_{g}+d_{h}-2}{d_{g} d_{h}}} .
$$

The $\mathrm{ABC}$ index is an excellent valuable index in the formation of heat in alkanes $[25,26]$.

Definition 1. For a graph $\xi$, the SK index [27] can be computed by

$$
S K(\xi)=\sum_{g h \in E(\xi)} \frac{d_{g}+d_{h}}{2} .
$$

Let $d_{g}$ and $d_{h}$ be the degrees of nodes $g$ and $h$ in $\xi$, respectively.

Definition 2. For a graph $\xi$, the $\mathrm{SK}_{1}$ index can be computed by

$$
S K_{1}(\xi)=\sum_{g h \in E(\xi)} \frac{d_{g} d_{h}}{2} .
$$

Let $d_{g}$ and $d_{h}$ be the degrees of nodes $g$ and $h$ in $\xi$, respectively.

Definition 3. For a graph $\xi$, the $\mathrm{SK}_{2}$ index can be computed by

$$
S K_{2}(\xi)=\sum_{g h \in E(\xi)}\left[\frac{d_{g}+d_{h}}{2}\right]^{2} .
$$

Let $d_{g}$ and $d_{h}$ be the degrees of nodes $g$ and $h$ in $\xi$, respectively.

In 2009, B. Lučić proposed the sum-connectivity index $(\chi)$ in [28] calculated by

$$
\chi_{-(1 / 2)}(G)=\sum_{g h \in E(G)}\left[d_{g}+d_{h}\right]^{-1 / 2} \text {. }
$$

In 2010, B. Zhou and Trinajstić furnished an index named general sum-connectivity index in $[24,29]$ and formulated as follows:

$$
\chi_{k}(\xi)=\sum_{g h \in E(\xi)}\left[d_{g}+d_{h}\right]^{k} .
$$

In 2005, X. Li and J. Zheng produced the generalized form of the first Zagreb index by calling it the "first general Zagreb index." The first general Zagreb index [30-35] of a graph $\xi$ was computed by ${ }^{k} M_{1}(\xi)=\sum_{g h \in E(\xi)}\left[d_{g}^{k-1}+d_{h}^{k-1}\right] ; k$ belongs to $R$, and $k \neq 0$ and $k \neq 1$.

In 2015, Boris Furtula and Ivan Gutman discovered an index named as "forgotten topological index" [36-38] and computed as

$$
F(G)=\sum_{g h \in E(G)}\left[d_{g}^{2}+d_{h}^{2}\right]
$$

\section{Topological Indices on Certain Chemical Graphs}

In this part of the research paper, we will compute the topological indices (degree based) such as ordinary generalized geometric-arithmetic (OGA) index, first and second Gourava indices, first and second hyper-Gourava indices, general Randic index $R_{\gamma}(\xi)$, for $\gamma=\{ \pm 1, \pm 1 / 2\}$, harmonic index, general version of harmonic index, atom-bond connectivity $(\mathrm{ABC})$ index, $\mathrm{SK}, \mathrm{SK}_{1}$, and $\mathrm{SK}_{2}$ indices, sumconnectivity index, general sum-connectivity index, first general Zagreb index, and forgotten topological indices for various types of chemical networks such as subdivided 
polythiophene network, subdivided hexagonal network, subdivided backbone DNA network, and subdivided honeycomb network.

2.1. Results for the Subdivided Polythiophene Network. Polythiophenes are rings with five elements having one heteroatom together with their benzo and other carbocylic. Polythiophene is used in electronic devices such as water purification devices, biosensors, and light-emitting diodes and in hydrogen storage [39]. In a subdivided polythiophene network, shown in Figure 1, we insert another vertex (degree 2) in every edge of $\xi$. In this way, we get a subdivided polythiophene network. In this section, we compute the subdivided polythiophene network using the above-defined topological indices. In the subdivided polythiophene network SPLY $n$, we have the number of nodes $11 n-1$ and edges $12 n-2$. A subdivided polythiophene network for $n=5$ is shown in Figure 1. We get two kinds of edges (degree based) that are $(2,2)$ and $(2,3)$. Table 1 gives us two types of edges. A subdivided polythiophene network SPLY 5 is displayed in Figure 1.

Theorem 1. For the subdivided polythiophene network, $S P L Y_{n}$, the ordinary generalized geometric-arithmetic index is calculated by

$$
\mathrm{OGA}_{k}(\xi)=6 n\left\{1+\left[\frac{\sqrt{24}}{5}\right]^{k}\right\}+\left\{4-6\left[\frac{\sqrt{24}}{5}\right]^{k}\right\}
$$

Proof. By letting $\xi$ as a subdivided polythiophene network SPLY $_{n}$, from Table 1, we know

$$
\begin{aligned}
& \operatorname{OGA}_{k}(\xi)=\sum_{g h \in E(\xi)}\left[\frac{\sqrt{4 d_{g} d_{h}}}{d_{g}+d_{h}}\right]^{k}, \\
& \operatorname{OGA}_{k}(\xi)=(6 n+4)\left[\frac{\sqrt{16}}{2+2}\right]^{k}+(6 n-6)\left[\frac{\sqrt{24}}{5}\right]^{k},
\end{aligned}
$$

and by doing some calculations, we get

$$
\mathrm{OGA}_{k}(\xi)=6 n\left[1+\left(\frac{\sqrt{24}}{5}\right)^{k}\right]+\left[4-6\left(\frac{\sqrt{24}}{5}\right)^{k}\right]
$$

Theorem 2. For the subdivided polythiophene network, $S P L Y_{n}$, the first and second Gourava indices are calculated by $G O_{1}(\xi)=114 n-34$ and $G_{2}(\xi)=276 n-116$.

Proof. By letting $\xi$ as a subdivided polythiophene network $\operatorname{SPLY}_{n}$, from Table 1, we know

$$
\begin{aligned}
& G O_{1}(\xi)=\sum_{g h \in E(\xi)}\left[\left(d_{g}+d_{h}\right)+\left(d_{g} d_{h}\right)\right], \\
& G O_{2}(\xi)=\sum_{g h \in E(\xi)}\left[\left(d_{g}+d_{h}\right)+\left(d_{g} d_{h}\right)\right],
\end{aligned}
$$

and by doing some calculations, we get

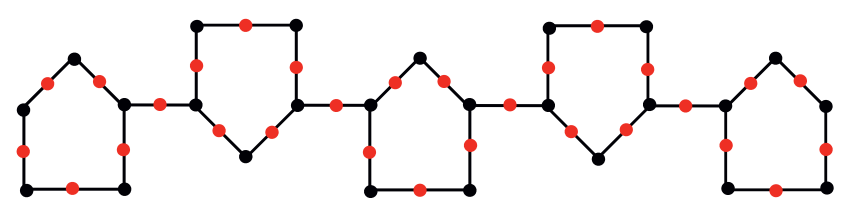

FIgURE 1: SPLY .

TABLE 1: Division of edges of a graph $\xi$ found on the degree of terminating nodes of each of the edges.

\begin{tabular}{lc}
\hline$\left(d_{g}, d_{h}\right)$ for $g h \in E(\xi)$ & Number of $E(\xi)$ \\
\hline$(2,2)$ & $6 n+4$ \\
$(2,3)$ & $6(n-1)$ \\
\hline & \\
$\mathrm{GO}_{1}(\xi)=(6 n+4)[(4)+(4)]+(6 n-6)[(5)+(6)]=114 n-34$, \\
$\mathrm{GO}_{2}(\xi)=(6 n+4)[16]+(6 n-6)[30]=276 n-116$.
\end{tabular}

Theorem 3. For the subdivided polythiophene network, $S P L Y_{n}$, the first and second hyper-Gourava indices are calculated by $H_{G O}(\xi)=1110 n-470$ and $\mathrm{HGO}_{2}(\xi)=6936 n-4376$.

Proof. By letting $\xi$ as a subdivided polythiophene network, SPLY $_{n}$, from Table 1, we know

$$
\begin{aligned}
& \mathrm{HGO}_{1}(\xi)=\sum_{g h \in E(\xi)}\left[\left(d_{g}+d_{h}\right)+\left(d_{g} d_{h}\right)\right]^{2}, \\
& \mathrm{HGO}_{2}(\xi)=\sum_{g h \in E(\xi)}\left[\left(d_{g}+d_{h}\right)+\left(d_{g} d_{h}\right)\right]^{2},
\end{aligned}
$$

and by doing some calculations, we get $\mathrm{HGO}_{1}(\xi)=(6 n+4)[64]+(6 n-6)[121]=1110 n-470$, and

$$
\mathrm{HGO}_{2}(\xi)=(6 n+4)[256]+(6 n-6)[900]=6936 n-4376 .
$$

Theorem 4. For the subdivided polythiophene network, $S P L Y_{n}$, the general Randic' index is calculated by

$$
R_{\gamma}\left(\operatorname{SPLY}_{n}\right)= \begin{cases}\frac{5}{2} n, & \text { for } \gamma=-1, \\ n\left(\frac{109}{20}\right)+\left(\frac{8}{5}\right), & \text { for } \gamma=-\frac{1}{2} \\ n\left(\frac{267}{10}\right)-\left(\frac{67}{10}\right), & \text { for } \gamma=\frac{1}{2} \\ 60 n-20, & \text { for } \gamma=1 .\end{cases}
$$

Proof. By letting $\xi$ as a subdivided polythiophene network SPLY $_{n}$ of $n$ dimensions, we have the number of nodes and 
edges in $\operatorname{SPLY}_{n}$ as $\left|V\left(\operatorname{SPLY}_{n}\right)\right|=11 n-1$ and $\left|E\left(\operatorname{SPLY}_{n}\right)\right|=12 n-2$, respectively.

We know that

$$
R_{\gamma}(\xi)=\sum_{g h \in E(\xi)}\left(d_{g} \cdot d_{h}\right)^{\gamma},
$$

for $\gamma=\{-1,1,-1 / 2,1 / 2\}$.

Case 1: if $\gamma=-1$, the application of Randic' index $R_{\gamma}(\xi)$

$$
R_{-1}(\xi)=\sum_{g h \in E(\xi)} \frac{1}{d_{g} d_{h}}
$$

using (23). From Table 1, we know $R_{-1}(\xi)=(6 n+4)(4)^{-1}+(6 n-6)(6)^{-1}$. By doing some calculations, we get $R_{-1}(\xi)=(5 / 2) n$.

Case 2: if $\gamma=-(1 / 2)$, the application of Randic' index $R_{\gamma}(\xi)$

$$
R_{-(1 / 2)}(\xi)=\sum_{g h \in E(\xi)} \frac{1}{\sqrt{\left(d_{g} \cdot d_{h}\right)}},
$$

using (23),

$$
R_{-(1 / 2)}(\xi)=(6 n+4) \frac{1}{\sqrt{4}}+(6 n-6) \frac{1}{\sqrt{6}},
$$

and by doing some calculations, we get $R_{-(1 / 2)}(\xi)=n(109 / 20)+(8 / 5)$.

Case 3: if $\gamma=(1 / 2)$, the application of Randic' index $R_{\gamma}(\xi)$

$$
R_{(1 / 2)}(\xi)=\sum_{g h \in E(\xi)} \sqrt{d_{g} \cdot d_{h}},
$$

using (23),

$$
R_{(1 / 2)}(\xi)=(6 n+4) \sqrt{4}+(6 n-6) \sqrt{6},
$$

and by doing some calculations, we get $R_{(1 / 2)}(\xi)=n(267 / 10)-(67 / 10)$.

Case 4: if $\gamma=1$, the application of Randic' index $R_{\gamma}(\xi)$

$$
R_{1}(\xi)=\sum_{g h \in E(\xi)}\left(d_{g} \cdot d_{h}\right)^{1},
$$

using (23),

$$
R_{1}(\xi)=(6 n+4)(4)+(6 n-6)(6),
$$

and by doing some calculations, we get $R_{1}(\xi)=60 n-20$.

Theorem 5. For the subdivided polythiophene network, $S P L Y_{n}$, the harmonic index is calculated by

$$
\mathrm{HI}(\xi)=\frac{2 n-2}{5} .
$$

Proof. By letting $\xi$ as a subdivided polythiophene network SPLY $_{n}$, from Table 1, we know

$$
\mathrm{HI}(\xi)=\sum_{g h \in E(\xi)} \frac{2}{d_{g}+d_{h}},
$$

and by doing some calculations, we get

$$
\mathrm{HI}(\xi)=(6 n+4)\left[\frac{1}{2}\right]+(6 n-6)\left[\frac{2}{5}\right]=\frac{2}{5}(n-1) \text {. }
$$

Theorem 6. For the subdivided polythiophene network, $S P L Y_{n}$, the general version of the harmonic index is calculated by

$$
H_{k} I(\xi)=\frac{6 n\left\{5^{k}+4^{k}\right\}+4\left\{5^{k}\right\}-6\left\{4^{k}\right\}}{\left\{10^{k}\right\}} .
$$

Proof. By letting $\xi$ as a subdivided polythiophene network SPLY $_{n}$, from Table 1, we know

$$
H_{k} I(\xi)=\sum_{g h \in E(\xi)}\left[\frac{2}{d_{g}+d_{h}}\right]^{k},
$$

and by doing some calculations, we get

$$
\begin{aligned}
H_{k} I(\xi) & =(6 n+4)\left[\frac{2}{2+2}\right]^{k}+(6 n-6)\left[\frac{2}{2+3}\right]^{k}, \\
& =\frac{6 n\left(5^{k}+4^{k}\right)+4\left(5^{k}\right)-6\left(4^{k}\right)}{(10)^{k}} .
\end{aligned}
$$

Theorem 7. For the subdivided polythiophene network, $S P L Y_{n}$, the atom-bond connectivity index is calculated by

$$
A B C(\xi)=6 \sqrt{2} n-\sqrt{2} .
$$

Proof. By letting $\xi$ as a subdivided polythiophene network $\mathrm{SPLY}_{n}$, from Table 1, we know

$$
A B C(\xi)=\sum_{g h \in E(\xi)} \sqrt{\frac{d_{g}+d_{h}-2}{d_{g} d_{h}}},
$$

and by doing some calculations, we get

$$
\begin{aligned}
A B C(\xi) & =(6 n+4) \sqrt{\frac{2+2-2}{4}}+(6 n-6) \sqrt{\frac{2+3-2}{6}} \\
& =\sqrt{2}(6 n-1) .
\end{aligned}
$$

Theorem 8. For the subdivided polythiophene network, $S P L Y_{n}, S K, S K_{1}$, and $S K_{2}$ indices are calculated by $S K(\xi)=$ $27 n-7, \quad S K_{1}(\xi)=30 n-10$, and $S K_{2}(\xi)=(1 / 2) \quad(123 n$ $-43)$, respectively.

Proof. By letting $\xi$ as a subdivided polythiophene network $\mathrm{SPLY}_{n}$, from Table 1, we know 


$$
\begin{aligned}
& S K(\xi)=\sum_{g h \in E(\xi)}\left[\frac{d_{g}+d_{h}}{2}\right], \\
& S K_{1}(\xi)=\sum_{g h \in E(\xi)}\left[\frac{d_{g} d_{h}}{2}\right], \\
& S K_{2}(\xi)=\sum_{g h \in E(\xi)}\left[\frac{d_{g}+d_{h}}{2}\right]^{2},
\end{aligned}
$$

and by doing some calculations, we get

$$
\begin{aligned}
& S K(\xi)=(6 n+4)(2)+(6 n-6)\left(\frac{5}{2}\right)=27 n-7, \\
& S K_{1}(\xi)=(6 n+4)(2)+(6 n-6)(3)=30 n-10, \\
& S K_{2}(\xi)=(6 n+4)(4)+(6 n-6)\left(\frac{25}{4}\right)=\frac{1}{2}(123 n-43) .
\end{aligned}
$$

Theorem 9. For the subdivided polythiophene network, SPLY $Y_{n}$, the sum-connectivity index is calculated by

$$
\chi_{-(1 / 2)}(\xi)=n\left\{3+\frac{6}{\sqrt{5}}\right\}+\left\{2-\frac{6}{\sqrt{5}}\right\} .
$$

Proof. By letting $\xi$ as a subdivided polythiophene network SPLY $_{n}$, from Table 1, we know

$$
\begin{aligned}
& \chi_{-(1 / 2)}(\xi)=\sum_{g h \in E(\xi)}\left[d_{g}+d_{h}\right]^{-(1 / 2)}, \\
& \chi_{-(1 / 2)}(\xi)=(6 n+4)\left(\frac{1}{2}\right)+(6 n-6)\left(\frac{1}{\sqrt{5}}\right),
\end{aligned}
$$

and by doing some calculations, we get

$$
\chi_{-(1 / 2)}(\xi)=n\left\{3+\frac{6}{\sqrt{5}}\right\}+\left\{2-\frac{6}{\sqrt{5}}\right\} \text {. }
$$

Theorem 10. For the subdivided polythiophene network, $S P L Y_{n}$, the general sum-connectivity index is calculated by

$$
\chi_{k}(\xi)=6 n\left\{5^{k}+4^{k}\right\}+4\left\{4^{k}\right\}-6\left\{5^{k}\right\} \text {. }
$$

Proof. By letting $\xi$ as a subdivided polythiophene network SPLY $_{n}$, from Table 1, we know

$$
\begin{aligned}
& \chi_{k}(\xi)=\sum_{g h \in E(\xi)}\left[d_{g}+d_{h}\right]^{k}, \\
& \chi_{k}(\xi)=(6 n+4)\left(4^{k}\right)+(6 n-6)\left(5^{k}\right),
\end{aligned}
$$

and by doing some calculations, we get

$$
\chi_{k}(\xi)=6 n\left(5^{k}+4^{k}\right)+4\left(4^{k}\right)-6\left(5^{k}\right)
$$

Theorem 11. For the subdivided polythiophene network, $S P L Y_{n}$, the first general Zagreb index is calculated by

$$
{ }^{k} M_{1}(\xi)=9 n\left\{2^{k}\right\}+2 n\left\{3^{k}\right\}+2\left\{\frac{2^{k}}{2}-3^{k}\right\} .
$$

Proof. By letting $\xi$ as a subdivided polythiophene network SPLY $_{n}$, from Table 1, we know

$$
\begin{aligned}
& { }^{k} M_{1}(\xi)=\sum_{g h \in E(\xi)}\left[d_{g}^{k-1}+d_{h}^{k-1}\right], \quad k>1, \\
& { }^{k} M_{1}(\xi)=(6 n+4)\left(2^{k}\right)+(6 n-6)\left(2^{k-1}+3^{k-1}\right),
\end{aligned}
$$

and by doing some calculations, we get

$$
{ }^{k} M_{1}(\xi)=9 n\left\{2^{k}\right\}+2 n\left\{3^{k}\right\}+2\left\{\frac{2^{k}}{2}-3^{k}\right\} \text {. }
$$

Theorem 12. For the subdivided polythiophene network, $S P L Y_{n}$, the forgotten index is calculated by

$$
F(\xi)=2\{63 n-23\} .
$$

Proof. By letting $\xi$ as a subdivided polythiophene network SPLY $_{n}$, from Table 1, we know

$$
\begin{aligned}
& F(\xi)=\sum_{g h \in E(\xi)}\left[d_{g}^{2}+d_{h}^{2}\right], \\
& F(\xi)=(6 n+4)(8)+(6 n-6)(13),
\end{aligned}
$$

and by doing some calculations, we get

$$
F(\xi)=126 n-46 .
$$

2.2. Results for the Subdivided Hexagonal Network. We construct a subdivided hexagonal network shown in Figure 2 by adding a new vertex in each edge. For this process, a triangular tiling is used. In this way, an $n$-dimensional subdivided hexagonal network is obtained and denoted by $\mathrm{SHX}_{n}$. A subdivided hexagonal network for $n=6$ is shown in Figure 2, whereas $n$ shows the number of nodes. The order of $\mathrm{SHX}_{n}$ is $12 n^{2}-18 n+7$ for $n>1$, and the size is $18 n^{2}-30 n+12$ for $n>1$. After the subdivision of this network, we have three types of edges that are $(2,3),(2,4)$, and $(2,6)$. The division of edges is shown in Table 2 . A subdivided hexagonal network $\mathrm{SHX}_{6}$ is displayed in Figure 2 .

Theorem 13. For the subdivided hexagonal network, $S H X_{n}$, the ordinary generalized geometric-arithmetic index is calculated by 


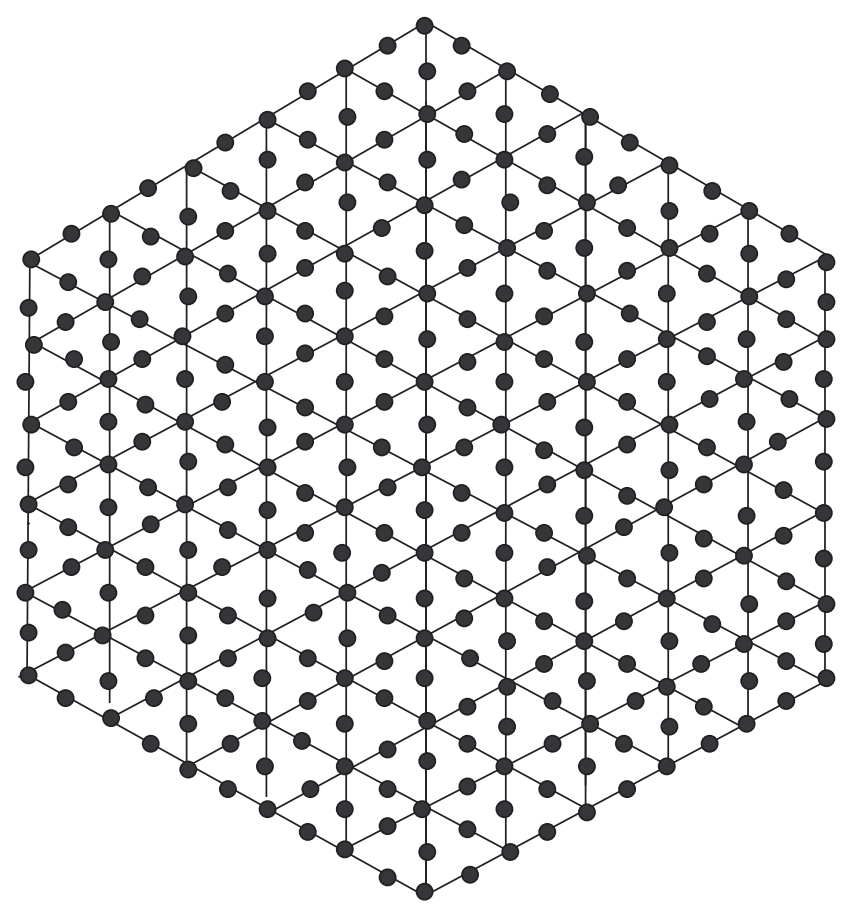

Figure 2: $\mathrm{SHX}_{6}$.

TABle 2: Division of edges of a graph $\xi$ found on the degree of terminating nodes of each of the edges.

\begin{tabular}{lc}
\hline$\left(d_{g}, d_{h}\right)$ for $g h \in E(\xi)$ & Number of $E(\xi)$ \\
\hline$(2,3)$ & 18 \\
$(2,4)$ & $24(n-2)$ \\
$(2,6)$ & $6\left(3 n^{2}-9 n+7\right)$ \\
\hline
\end{tabular}

$$
\begin{aligned}
\operatorname{OGA}_{k}(\xi)= & 18 n^{2}\left\{\frac{\sqrt{48}}{8}\right\}^{k}+n\left[24\left\{\frac{\sqrt{32}}{6}\right\}^{k}-54\left\{\frac{\sqrt{48}}{8}\right\}^{k}\right] \\
& +\left[42\left\{\frac{\sqrt{48}}{8}\right\}^{k}-48\left\{\frac{\sqrt{32}}{6}\right\}^{k}\right] .
\end{aligned}
$$

Proof. By letting 1 as a subdivided hexagonal network $\mathrm{SHX}_{n}$, from Table 2, we know

$$
\begin{aligned}
\operatorname{OGA}_{k}(\xi)= & \sum_{g h \in E(\xi)}\left[\frac{\sqrt{4 d_{g} d_{h}}}{d_{g}+d_{h}}\right]^{k}, \\
\operatorname{OGA}_{k}(\xi)= & (18)\left[\frac{\sqrt{24}}{5}\right]^{k}+24(n-2)\left[\frac{\sqrt{32}}{6}\right]^{k} \\
& +6\left(3 n^{2}-9 n+7\right)\left\{\frac{\sqrt{48}}{8}\right\}^{k},
\end{aligned}
$$

$$
\begin{aligned}
\operatorname{OGA}_{k}(\xi)= & 18 n^{2}\left\{\frac{\sqrt{48}}{8}\right\}^{k}+n\left[24\left\{\frac{\sqrt{32}}{6}\right\}^{k}-54\left\{\frac{\sqrt{48}}{8}\right\}^{k}\right] \\
& +\left[42\left\{\frac{\sqrt{48}}{8}\right\}^{k}-48\left\{\frac{\sqrt{32}}{6}\right\}^{k}\right]
\end{aligned}
$$

Theorem 14. For the subdivided hexagonal network, $S H X_{n}$, the first and second Gourava indices are calculated by

$$
\begin{aligned}
& G O_{1}(\xi)=360 n^{2}-744 n+366 \\
& G O_{2}(\xi)=1728 n^{2}-4032 n+2268
\end{aligned}
$$

Proof. By letting $\xi$ as a subdivided hexagonal network $\mathrm{SHX}_{n}$, from Table 2, we know

$$
\begin{aligned}
& G O_{1}(\xi)=\sum_{g h \in E(\xi)}\left[\left(d_{g}+d_{h}\right)+\left(d_{g} d_{h}\right)\right], \\
& G_{2}(\xi)=\sum_{g h \in E(\xi)}\left[\left(d_{g}+d_{h}\right)+\left(d_{g} d_{h}\right)\right],
\end{aligned}
$$

and by doing some calculations, we get

$$
\begin{aligned}
G O_{1}(\xi)= & (18)[(5)+(6)]+24(n-2)[(6)+(8)] \\
& +6\left(3 n^{2}-9 n+7\right)[(8)+(12)] \\
= & 360 n^{2}-744 n+366, \\
G_{2}(\xi)= & (18)[30]+24(n-2)[48] \\
& +6\left(3 n^{2}-9 n+7\right)[96], \\
= & 1728 n^{2}-4032 n+2268 .
\end{aligned}
$$

Theorem 15. For the subdivided hexagonal network, $S H X_{n}$, the first and second hyper-Gourava indices are calculated by $\mathrm{HGO}_{1}(\xi)=7200 n^{2}-16896 n+9570$ and $\mathrm{HGO}_{2}(\xi)=$ $165888 n^{2}-442368 n+292680$.

Proof. By letting $\xi$ as a subdivided hexagonal network $\mathrm{SHX}_{n}$, from Table 2, we know

$$
\begin{aligned}
& \operatorname{HGO}_{1}(\xi)=\sum_{g h \in E(\xi)}\left[\left(d_{g}+d_{h}\right)+\left(d_{g} d_{h}\right)\right]^{2}, \\
& \mathrm{HGO}_{2}(\xi)=\sum_{g h \in E(\xi)}\left[\left(d_{g}+d_{h}\right)+\left(d_{g} d_{h}\right)\right]^{2},
\end{aligned}
$$

and by doing some calculations, we get

$$
\begin{aligned}
\mathrm{HGO}_{1}(\xi)= & (18)[121]+24(n-2)[196] \\
& +6\left(3 n^{2}-9 n+7\right)[400] \\
= & 7200 n^{2}-16896 n+9570, \\
\mathrm{HGO}_{2}(\xi)= & (18)[900]+24(n-2)[2304] \\
& +6\left(3 n^{2}-9 n+7\right)[9216], \\
= & 165888 n^{2}-442368 n+292680 .
\end{aligned}
$$

and by doing some calculations, we get 
Theorem 16. For the subdivided hexagonal network, $S H X_{n}$, $n>1$, the general Randic' index is calculated by

$$
R_{\gamma}\left(\mathrm{SHX}_{n}\right)= \begin{cases}\frac{3 n^{2}-3 n+1}{2}, & \text { for } \gamma=-1, \\ 3 n^{2} \sqrt{3}+n\{\sqrt{72}-9 \sqrt{3}\}+2.501, & \text { for } \gamma=-\frac{1}{2}, \\ 62.35 n^{2}-119.17 n+53.82, & \text { for } \gamma=\frac{1}{2}, \\ 216 n^{2}-456 n+228, & \text { for } \gamma=1 .\end{cases}
$$

Proof. By letting $\xi$ as a subdivided hexagonal network $\mathrm{SHX}_{n}$ of $n$ dimensions, we have the number of nodes and edges in $\mathrm{SHX}_{n}$ as $\left|V\left(\mathrm{SHX}_{n}\right)\right|=12 n^{2}-18 \mathrm{~N}+7$ for $n>1$ and $\left|E\left(\mathrm{SHX}_{n}\right)\right|=18 n^{2}-30 n+12$ for $n>1$, respectively. We know that

$$
R_{\gamma}(\xi)=\sum_{g h \in E(\xi)}\left(d_{g} \cdot d_{h}\right)^{\gamma}
$$

for $\gamma=\{-1,1,-1 / 2,1 / 2\}$.

Case 1: if $\gamma=-1$, the application of Randic' index $R_{\gamma}(\xi)$

$$
R_{-1}(\xi)=\sum_{g h \in E(\xi)} \frac{1}{d_{g} d_{h}},
$$

using (63). From Table 2, we know

$$
\begin{aligned}
R_{-1}(\xi)= & (18)(6)^{-1}+24(n-2)(8)^{-1} \\
& +6\left(3 n^{2}-9 n+7\right)(12)^{-1}
\end{aligned}
$$

By doing some calculations, we get $R_{-1}(\xi)=\left(3 n^{2}-3 n+1 / 2\right)$

Case 2: if $\gamma=-(1 / 2)$, the application of Randic' index $R_{\gamma}(\xi)$

$$
R_{-(1 / 2)}(\xi)=\sum_{g h \in E(\xi)} \frac{1}{\sqrt{\left(d_{g} \cdot d_{h}\right)}}
$$

using (63),

$$
R_{-(1 / 2)}(\xi)=(18) \frac{1}{\sqrt{6}}+24(n-2) \frac{1}{\sqrt{8}}+6\left(3 n^{2}-9 n+7\right) \frac{1}{\sqrt{12}}
$$

By doing some calculations, we get

$$
R_{-(1 / 2)}(\xi)=3 n^{2} \sqrt{3}+n\{\sqrt{72}-9 \sqrt{3}\}+2.501 .
$$

Case 3: if $\gamma=(1 / 2)$, the application of Randic' index $R_{\gamma}(\xi)$

$$
R_{(1 / 2)}(\xi)=\sum_{g h \in E(\xi)} \sqrt{d_{g} \cdot d_{h}}
$$

using (63),

$$
R_{(1 / 2)}(\xi)=(18) \sqrt{6}+24(n-2) \sqrt{8}+6\left(3 n^{2}-9 n+7\right) \sqrt{12}
$$

By doing some calculations, we get

$$
R_{(1 / 2)}(\xi)=62.35 n^{2}-119.17 n+53.82 .
$$

Case 4: if $\gamma=1$, the application of Randic' index $R_{\gamma}(\xi)$

$$
R_{1}(\xi)=\sum_{g h \in E(\xi)}\left(d_{g} \cdot d_{h}\right)^{1},
$$

using (63),

$R_{1}(\xi)=(18)(6)+24(n-2)(8)+6\left(3 n^{2}-9 n+7\right)(12)$.

By doing some calculations, we get

$$
R_{1}(\xi)=216 n^{2}-456 n+228
$$

Theorem 17. For the subdivided hexagonal network, $S H X_{n}$, the harmonic index is calculated by

$$
H I(\xi)=4.5 n^{2}-5.5 n+1.7 .
$$

Proof. By letting $\xi$ as a subdivided hexagonal network $\mathrm{SHX}_{n}$, from Table 2, we know

$$
H I(\xi)=\sum_{g h \in E(\xi)} \frac{2}{d_{g}+d_{h}} .
$$

By doing some calculations, we get

$$
\begin{aligned}
H I(\xi) & =(18)\left[\frac{2}{5}\right]+24(n-2)\left[\frac{2}{6}\right]+6\left(3 n^{2}-9 n+7\right)\left[\frac{2}{8}\right], \\
& =\frac{1}{10}\left(45 n^{2}-55 n+17\right)=4.5 n^{2}-5.5 n+1.7
\end{aligned}
$$

Theorem 18. For the subdivided hexagonal network, $S H X_{n}$, the general version of the harmonic index is calculated by

$$
\begin{aligned}
H_{k} I(\xi)= & 18 n^{2}\left\{4^{-k}\right\}+2 n\left\{12\left\{3^{-k}\right\}-27\left\{4^{-k}\right\}\right\} \\
& +\left[18\left\{2^{k}\left\{5^{-k}\right\}\right\}-48\left\{3^{-k}\right\}+42\left\{4^{-k}\right\}\right] .
\end{aligned}
$$

Proof. By letting $\xi$ as a subdivided hexagonal network $\mathrm{SHX}_{n}$, from Table 2, we know

$$
H_{k} I(\xi)=\sum_{g h \in E(\xi)}\left[\frac{2}{d_{g}+d_{h}}\right]^{k} .
$$

By doing some calculations, we get 


$$
\begin{aligned}
H_{k} I(\xi)= & (18)\left[\frac{2}{2+3}\right]^{k}+24(n-2)\left[\frac{2}{2+4}\right]^{k} \\
& +6\left(3 n^{2}-9 n+7\right)\left[\frac{2}{2+6}\right]^{k} \\
= & n^{2}\left(\frac{18}{4^{k}}\right)+n\left(\frac{24}{3^{k}}-\frac{54}{4^{k}}\right)+\left(18\left(\frac{2^{k}}{5^{k}}\right)-\frac{48}{3^{k}}+\frac{42}{4^{k}}\right) \\
= & 18 n^{2}\left\{4^{-k}\right\}+2 n\left\{12\left\{3^{-k}\right\}-27\left\{4^{-k}\right\}\right\} \\
& +\left[18\left\{2^{k}\left\{5^{-k}\right\}\right\}-48\left\{3^{-k}\right\}+42\left\{4^{-k}\right\}\right] .
\end{aligned}
$$

Theorem 19. For the subdivided hexagonal network, $S H X_{n}$, the atom-bond connectivity index is calculated by

$$
A B C(\xi)=9 \sqrt{2} n^{2}-15 \sqrt{2} n+6 \sqrt{2} .
$$

Proof. By letting $\xi$ as a subdivided hexagonal network $\mathrm{SHX}_{n}$, from Table 2, we know

$$
A B C(\xi)=\sum_{g h \in E(\xi)} \sqrt{\frac{d_{g}+d_{h}-2}{d_{g} d_{h}}},
$$

and by doing some calculations, we get

$$
\begin{aligned}
A B C(\xi)= & (18) \sqrt{\frac{2+3-2}{6}}+24(n-2) \sqrt{\frac{2+4-2}{8}} \\
& +6\left(3 n^{2}-9 n+7\right) \sqrt{\frac{2+6-2}{12}} \\
= & 9 \sqrt{2} n^{2}-15 \sqrt{2} n+6 \sqrt{2} .
\end{aligned}
$$

Theorem 20. For the subdivided hexagonal network, $S H X_{n}$, $S K, S K_{1}$, and $S K_{2}$ indices are calculated by $S K(\xi)=72 n^{2}-$ $144 n+69, S K_{1}(\xi)=108 n^{2}-228 n+498$, and $S K_{2}(\xi)=$ $288 n^{2}-648 n+7052$, respectively.

Proof. By letting $\xi$ as a subdivided hexagonal network $\mathrm{SHX}_{n}$, from Table 2, we know

$$
\begin{aligned}
& S K(\xi)=\sum_{g h \in E(\xi)}\left[\frac{d_{g}+d_{h}}{2}\right], \\
& S K_{1}(\xi)=\sum_{g h \in E(\xi)}\left[\frac{d_{g} d_{h}}{2}\right], \\
& S K_{2}(\xi)=\sum_{g h \in E(\xi)}\left[\frac{d_{g}+d_{h}}{2}\right]^{2},
\end{aligned}
$$

and by doing some calculations, we get

$$
\begin{aligned}
S K(\xi) & =(18)\left(\frac{5}{2}\right)+24(n-2)\left(\frac{6}{2}\right)+6\left(3 n^{2}-9 n+7\right)\left(\frac{8}{2}\right) \\
& =72 n^{2}-144 n+69 . \\
S K_{1}(\xi) & =18(3)+24(n-2)(4)+36\left(3 n^{2}-9 n+7\right) \\
& =108 n^{2}-228 n+498 . \\
S K_{2}(\xi) & =(18)\left(\frac{25}{4}\right)+24(n-2)(9)+6\left(3 n^{2}-9 n+7\right)(16 \\
& =288 n^{2}-648 n+\frac{705}{2} .
\end{aligned}
$$

Theorem 21. For the subdivided hexagonal network, $S H X_{n}$, the sum-connectivity index is calculated by

$$
\chi_{-(1 / 2)}(\xi)=18 n^{2}\left\{\frac{1}{\sqrt{8}}\right\}+n\left\{\frac{24}{\sqrt{6}}-\frac{54}{\sqrt{8}}\right\}+\left\{\frac{18}{\sqrt{5}}-\frac{48}{\sqrt{6}}+\frac{42}{\sqrt{8}}\right\} .
$$

Proof. By letting $\xi$ as a subdivided hexagonal network $\mathrm{SHX}_{n}$, from Table 2, we know

$$
\begin{aligned}
\chi_{-(1 / 2)}(\xi)= & \sum_{g h \in E(\xi)}\left[d_{g}+d_{h}\right]^{-(1 / 2)} \\
\chi_{-(1 / 2)}(\xi)= & (18)\left(\frac{1}{\sqrt{6}}\right)+24(n-2)\left(\frac{1}{\sqrt{8}}\right) \\
& +6\left(3 n^{2}-9 n+7\right)\left(\frac{1}{\sqrt{12}}\right)
\end{aligned}
$$

and by doing some calculations, we get

$$
\begin{aligned}
\chi_{-(1 / 2)}(\xi) & =n^{2}\left(\frac{18}{\sqrt{8}}\right)+n\left(\frac{24}{\sqrt{6}}-\frac{54}{\sqrt{8}}\right)+\left(\frac{18}{\sqrt{5}}-\frac{48}{\sqrt{6}}+\frac{42}{\sqrt{8}}\right) \\
& =18 n^{2}\left(\frac{1}{\sqrt{8}}\right)+n\left(\frac{24}{\sqrt{6}}-\frac{54}{\sqrt{8}}\right)+\left(\frac{18}{\sqrt{5}}-\frac{48}{\sqrt{6}}+\frac{42}{\sqrt{8}}\right) .
\end{aligned}
$$

Theorem 22. For the subdivided hexagonal network, $S H X_{n}$, the general sum-connectivity index is calculated by

$$
\begin{aligned}
\chi_{k}(\xi)= & 18 n^{2}\left\{8^{k}\right\}+n\left\{24\left(6^{k}\right)-54\left(8^{k}\right)\right\} \\
& +\left\{18\left(5^{k}\right)-48\left(6^{k}\right)+42\left(8^{k}\right)\right\} .
\end{aligned}
$$

Proof. By letting $\xi$ as a subdivided hexagonal network $\mathrm{SHX}_{n}$, from Table 2, we know 


$$
\begin{aligned}
& \chi_{k}(\xi)=\sum_{g h \in E(\xi)}\left[d_{g}+d_{h}\right]^{k}, \\
& \chi_{k}(\xi)=(18)\left(5^{k}\right)+24(n-2)\left(6^{k}\right)+6\left(3 n^{2}-9 n+7\right)\left(8^{k}\right),
\end{aligned}
$$

and by doing some calculations, we get

$$
\begin{aligned}
\chi_{k}(\xi)== & 18 n^{2}\left\{8^{k}\right\}+n\left\{24\left(6^{k}\right)-54\left(8^{k}\right)\right\} \\
& +\left\{18\left(5^{k}\right)-48\left(6^{k}\right)+42\left(8^{k}\right)\right\} .
\end{aligned}
$$

Theorem 23. For the subdivided hexagonal network, $S H X_{n}$, the first general Zagreb index is calculated by

$$
\begin{aligned}
{ }^{k} M_{1}(\xi)= & n^{2}\left\{\left\{3\left\{6^{k}\right\}\right\}+9\left\{2^{k}\right\}\right\}+n\left\{6\left\{4^{k}\right\}-15\left\{2^{k}\right\}\right. \\
& \left.-9\left\{6^{k}\right\}\right\}+\left\{6\left\{2^{k}\right\}+6\left\{3^{k}\right\}-12\left\{4^{k}\right\}+7\left\{6^{k}\right\}\right\} .
\end{aligned}
$$

Proof. By letting $\xi$ as a subdivided hexagonal network $\mathrm{SHX}_{n}$, from Table 2, we know

$$
\begin{aligned}
{ }^{k} M_{1}(\xi)= & \sum_{g h \in E(\xi)}\left[d_{g}^{k-1}+d_{h}^{k-1}\right], \quad k>1, \\
{ }^{k} M_{1}(\xi)= & \{18\}\left(\frac{2^{k}}{2}+\frac{3^{k}}{3}\right)+\{24 n-48\}\left(\frac{2^{k}}{2}+\frac{4^{k}}{4}\right) \\
& +6\left(3 n^{2}-9 n+7\right)\left(2^{k-1}+6^{k-1}\right),
\end{aligned}
$$

and by doing some calculations, we get

$$
\begin{aligned}
{ }^{k} M_{1}(\xi)= & n^{2}\left\{36^{k}+92^{k}\right\}+n 64^{k}-152^{k}-96^{k} \\
& +\left\{62^{k}+63^{k}-124^{k}+76^{k}\right\} .
\end{aligned}
$$

Theorem 24. For the subdivided hexagonal network, $S H X_{n}$ the forgotten index is calculated by

$$
F(\xi)=2\left\{360 n^{2}-840 n+477\right\}
$$

Proof. By letting $\xi$ as a subdivided hexagonal network $\mathrm{SHX}_{n}$, from Table 2, we know

$$
\begin{aligned}
& F(\xi)=\sum_{g h \in E(\xi)}\left[d_{g}^{2}+d_{h}^{2}\right], \\
& F(\xi)=(18)(13)+24(n-2)(20)+6\left(3 n^{2}-9 n+7\right)(40),
\end{aligned}
$$

and by doing some calculations, we get

$$
F(\xi)=2\left\{360 n^{2}-840 n+477\right\} .
$$

2.3. Results for the Subdivided Backbone DNA Network. The structure of DNA is called a double helix as it is made of two strands that wind around each other that looks like a staircase [40]. Each strand has a backbone made of deoxyribose, sugar, and a phosphate group. These sugar and phosphates make up the backbone, while the nitrogen bases are found in the centre and hold the two strands together. There are 4 bases attached to each sugar which are adenine, cytosine, guanine, and thymine. Both ends of DNA have a number, i.e., one end is ' 5 and the other is ' 3 . In a subdivided backbone DNA network, shown in Figure 3, we insert another node (degree 2 ) in each edge of $\xi$. In this way, we get a subdivided backbone DNA network of $n$ dimensions. A subdivided backbone DNA network for $n=4$ is displayed in Figure 3. A subdivided backbone DNA network is symbolized as $\operatorname{SBB}_{\mathrm{DNA}}(n)$. The order and size of $\operatorname{SBB}_{\mathrm{DNA}}(n)$ are $15 n-5$ and $16 n-6$, respectively. We obtain two types of edges (degree based) that are $(2,2)$ and $(2,3)$. Table 3 gives us two kinds of edges. A subdivided backbone DNA network $\mathrm{SBB}_{\mathrm{DNA}}(4)$ is shown in Figure 3.

Theorem 25. For the subdivided backbone DNA network, $S B B_{D N A}(n)$, the ordinary generalized geometric-arithmetic index is calculated by

$$
\mathrm{OGA}_{k}(\xi)=2\left\{n\left[5+3\left\{\frac{\sqrt{24}}{5}\right\}^{k}\right]-3\left\{\frac{\sqrt{24}}{5}\right\}^{k}\right\}
$$

Proof. By letting $\xi$ as a subdivided backbone DNA network $\operatorname{SBB}_{\mathrm{DNA}}(n)$, from Table 3, we know

$$
\begin{aligned}
& \operatorname{OGA}_{k}(\xi)=\sum_{g h \in E(\xi)}\left[\frac{\sqrt{4 d_{g} d_{h}}}{d_{g}+d_{h}}\right]^{k}, \\
& \operatorname{OGA}_{k}(\xi)=10 n\left[\frac{\sqrt{16}}{2+2}\right]^{k}+(6 n-6)\left[\frac{\sqrt{24}}{5}\right]^{k},
\end{aligned}
$$

and by doing some calculations, we get

$$
\mathrm{OGA}_{k}(\xi)=2\left\{n\left[5+3\left\{\frac{\sqrt{24}}{5}\right\}^{k}\right]-3\left\{\frac{\sqrt{24}}{5}\right\}^{k}\right\} .
$$

Theorem 26. For the subdivided backbone DNA network, $S B B_{D N A}(n)$, the first and second Gourava indices are calculated by $G_{1}(\xi)=146 n-66$ and $G_{2}(\xi)=340 n-180$.

Proof. By letting $\xi$ as a subdivided backbone DNA network $\mathrm{SBB}_{\mathrm{DNA}}(n)$, from Table 3, we know

$$
\begin{aligned}
& \mathrm{GO}_{1}(\xi)=\sum_{g h \in E(\xi)}\left[\left(d_{g}+d_{h}\right)+\left(d_{g} d_{h}\right)\right], \\
& \mathrm{GO}_{2}(\xi)=\sum_{g h \in E(\xi)}\left[\left(d_{g}+d_{h}\right)+\left(d_{g} d_{h}\right)\right],
\end{aligned}
$$

and by doing some calculations, we get 


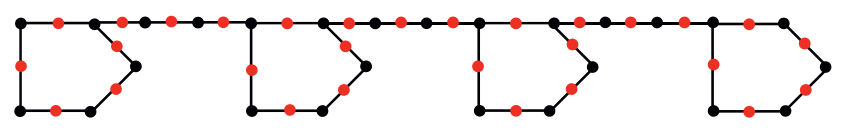

Figure 3: $\operatorname{SBB}_{\text {DNA }}(4)$.

Table 3: Division of edges of a graph $\xi$ found on the degree of terminating nodes of each of the edges.

\begin{tabular}{lc}
\hline$\left(d_{g}, d_{h}\right)$ for $g h \in E(\xi)$ & Number of $E(\xi)$ \\
\hline$(2,2)$ & $10 n$ \\
$(2,3)$ & $6(n-1)$ \\
\hline
\end{tabular}

$\mathrm{GO}_{1}(\xi)=10 n[(4)+(4)]+(6 n-6)[(5)+(6)]=146 n-66$,

$\mathrm{GO}_{2}(\xi)=10 n[16]+(6 n-6)[30]=340 n-180$.

Theorem 27. For the subdivided backbone DNA network, $S B B_{D N A}(n)$, the first and second hyper-Gourava indices are calculated by

$$
\begin{aligned}
& \mathrm{HGO}_{1}(\xi)=1366 n-726 \\
& \mathrm{HGO}_{2}(\xi)=7960 n-5400 .
\end{aligned}
$$

Proof. By letting $\xi$ as a subdivided backbone DNA network $\mathrm{SBB}_{\mathrm{DNA}}(n)$, from Table 3, we know

$$
\begin{aligned}
& \operatorname{HGO}_{1}(\xi)=\sum_{g h \in E(\xi)}\left[\left(d_{g}+d_{h}\right)+\left(d_{g} d_{h}\right)\right]^{2}, \\
& \mathrm{HGO}_{2}(\xi)=\sum_{g h \in E(\xi)}\left[\left(d_{g}+d_{h}\right)+\left(d_{g} d_{h}\right)\right]^{2},
\end{aligned}
$$

and by doing some calculations, we get

$$
\begin{aligned}
& \mathrm{HGO}_{1}(\xi)=10 n[64]+(6 n-6)[121]=1366 n-726 \\
& \mathrm{HGO}_{2}(\xi)=10 n[256]+(6 n-6)[900]=7960 n-5400
\end{aligned}
$$

Theorem 28. For the subdivided backbone DNA network, $S B B_{D N A}(n)$, the general Randic' index is calculated by

$$
R_{\gamma}\left(S B B_{D N A}(n)\right)= \begin{cases}\frac{7}{2} n-1, & \text { for } \gamma=-1, \\ n\left\{\frac{149}{20}\right\}-\left\{\frac{49}{20}\right\}, & \text { for } \gamma=-\frac{1}{2}, \\ n\left\{\frac{347}{10}\right\}-\left\{\frac{147}{10}\right\}, & \text { for } \gamma=\frac{1}{2}, \\ 76 n-36, & \text { for } \gamma=1 .\end{cases}
$$

Proof. By letting $\xi$ as a subdivided backbone DNA network $\mathrm{SBB}_{\mathrm{DNA}}(n)$ of $n$ dimensions, we have the order and size of $\xi$ in $\operatorname{SBB}_{\mathrm{DNA}}(n)$ as $\left|V\left(\operatorname{SBB}_{\mathrm{DNA}}(n)\right)\right|=15 n-5$ and $\left|E\left(\operatorname{SBB}_{\mathrm{DNA}}(n)\right)\right|=16 n-6$, respectively.
We know that

$$
R_{\gamma}(\xi)=\sum_{g h \in E(\xi)}\left(d_{g} \cdot d_{h}\right)^{\gamma}
$$

for $\gamma=\{-1,1,-1 / 2,1 / 2\}$.

Case 1: if $\gamma=-1$, the application of Randic' index $R_{\gamma}(\xi)$

$$
R_{-1}(\xi)=\sum_{g h \in E(\xi)} \frac{1}{d_{g} d_{h}}
$$

using (107). From Table 3, we know $R_{-1}(\xi)=10 n(4)^{-1}+(6 n-6)(6)^{-1}$. By doing some calculations, we get $R_{-1}(\xi)=(7 / 2) n-1$.

Case 2: if $\gamma=-(1 / 2)$, the application of Randic' index $R_{\gamma}(\xi)$

$$
R_{-(1 / 2)}(\xi)=\sum_{g h \in E(\xi)} \frac{1}{\sqrt{\left(d_{g} \cdot d_{h}\right)}}
$$

using (107),

$$
R_{-(1 / 2)}(\xi)=10 n \frac{1}{\sqrt{4}}+(6 n-6) \frac{1}{\sqrt{6}}
$$

and by doing some calculations, we get $R_{-(1 / 2)}(\xi)=n(149 / 20)-(49 / 20)$.

Case 3: if $\gamma=(1 / 2)$, the application of Randic' index $R_{\gamma}(\xi)$

$$
R_{(1 / 2)}(\xi)=\sum_{g h \in E(\xi)} \sqrt{d_{g} \cdot d_{h}}
$$

using (107)

$$
R_{(1 / 2)}(\xi)=10 n \sqrt{4}+(6 n-6) \sqrt{6}
$$

and by doing some calculations, we get $R_{(1 / 2)}(\xi)=n(347 / 10)-(147 / 10)$.

Case 4: if $\gamma=1$, the application of Randic' index $R_{\gamma}(\xi)$

$$
R_{1}(\xi)=\sum_{g h \in E(\xi)}\left(d_{g} \cdot d_{h}\right)^{1},
$$

using (107)

$$
R_{1}(\xi)=10 n(4)+(6 n-6)(6),
$$

and by doing some calculations, we get $R_{1}(\xi)=76 n-36$.

Theorem 29. For the subdivided backbone DNA network, $S B B_{D N A}(n)$, the harmonic index is calculated by

$$
H I(\xi)=\frac{37 n-12}{5}
$$

Proof. By letting $\xi$ as a subdivided backbone DNA network $\operatorname{SBB}_{\text {DNA }}(n)$, from Table 3 , we know 


$$
\mathrm{HI}(\xi)=\sum_{g h \in E(\xi)} \frac{2}{d_{g}+d_{h}},
$$

and by doing some calculations, we get

$$
H I(\xi)=10 n\left[\frac{1}{2}\right]+(6 n-6)\left[\frac{2}{5}\right]=\frac{37 n-12}{5} .
$$

Theorem 30. For the subdivided backbone DNA network, $S B B_{D N A}(n)$, the general version of the harmonic index is calculated by

$$
H_{k} I(\xi)=2 n\left\{\frac{5}{2^{k}}+\frac{3}{5^{k}} 2^{k}\right\}-\frac{6}{5^{k}} 2^{k} .
$$

Proof. By letting $\xi$ as a subdivided backbone DNA network $\operatorname{SBB}_{\mathrm{DNA}}(n)$, from Table 3, we know

$$
H_{k} I(\xi)=\sum_{g h \in E(\xi)}\left[\frac{2}{d_{g}+d_{h}}\right]^{k},
$$

and by doing some calculations, we get

$$
\begin{aligned}
H_{k} I(\xi) & =10 n\left[\frac{2}{2+2}\right]^{k}+(6 n-6)\left[\frac{2}{2+3}\right]^{k} \\
& =2 n\left\{\frac{5}{2^{k}}+\frac{3}{5^{k}} 2^{k}\right\}-\frac{6}{5^{k}} 2^{k} .
\end{aligned}
$$

Theorem 31. For the subdivided backbone DNA network, $S B B_{D N A}(n)$, the atom-bond connectivity index is calculated by

$$
A B C(\xi)=\frac{2\{8 n-3\}}{\sqrt{2}} .
$$

Proof. By letting $\xi$ as a subdivided backbone DNA network $\operatorname{SBB}_{\mathrm{DNA}}(n)$, from Table 3, we know

$$
A B C(\xi)=\sum_{g h \in E(\xi)} \sqrt{\frac{d_{g}+d_{h}-2}{d_{g} d_{h}}} .
$$

By doing some calculations, we get

$$
A B C(\xi)=10 n \sqrt{\frac{2+2-2}{4}}+(6 n-6) \sqrt{\frac{2+3-2}{6}}=\frac{2\{8 n-3\}}{\sqrt{2}} .
$$

Theorem 32. For the subdivided backbone DNA network, $S B B_{D N A}(n), S K, S K_{1}$, and $S K_{2}$ indices are calculated by $S K(\xi)=35 n-15, S K_{1}(\xi)=38 n-18$, and $S K_{2}(\xi)=(1 / 2)$ $(155 n-75)$, respectively.

Proof. By letting $\xi$ as a subdivided backbone DNA network $\operatorname{SBB}_{\mathrm{DNA}}(n)$, from Table 3, we know

$$
\begin{aligned}
& S K(\xi)=\sum_{g h \in E(\xi)}\left[\frac{d_{g}+d_{h}}{2}\right], \\
& S K_{1}(\xi)=\sum_{g h \in E(\xi)}\left[\frac{d_{g} d_{h}}{2}\right], \\
& S K_{2}(\xi)=\sum_{g h \in E(\xi)}\left[\frac{d_{g}+d_{h}}{2}\right]^{2} .
\end{aligned}
$$

By doing some calculations, we get

$$
\begin{aligned}
& S K(\xi)=10 n(2)+(6 n-6)\left(\frac{5}{2}\right)=35 n-15, \\
& S K_{1}(\xi)=10 n(2)+(6 n-6)(3)=38 n-18, \\
& S K_{2}(\xi)=10 n(4)+(6 n-6)\left(\frac{25}{4}\right)=\frac{1}{2}(155 n-75) .
\end{aligned}
$$

Theorem 33. For the subdivided backbone DNA network, $\mathrm{SBB}_{D N A}(n)$, the sum-connectivity index is calculated by

$$
\chi_{-(1 / 2)}(\xi)=n\left\{5+\frac{6}{\sqrt{5}}\right\}-\frac{6}{\sqrt{5}} \text {. }
$$

Proof. By letting $\xi$ as a subdivided backbone DNA network $\operatorname{SBB}_{\text {DNA }}(n)$, from Table 3 , we know

$$
\begin{aligned}
& \chi_{-(1 / 2)}(\xi)=\sum_{g h \in E(\xi)}\left[d_{g}+d_{h}\right]^{-(1 / 2)}, \\
& \chi_{-(1 / 2)}(\xi)=10 n\left(\frac{1}{2}\right)+(6 n-6)\left(\frac{1}{\sqrt{5}}\right) .
\end{aligned}
$$

By doing some calculations, we get

$$
\chi_{-(1 / 2)}(\xi)=n\left\{5+\frac{6}{\sqrt{5}}\right\}-\frac{6}{\sqrt{5}} .
$$

Theorem 34. For the subdivided backbone DNA network, $S B B_{D N A}(n)$, the general sum-connectivity index is calculated by

$$
\chi_{k}(\xi)=n\left\{10\left\{4^{k}\right\}+6\left\{5^{k}\right\}\right\}-6\left\{5^{k}\right\} .
$$

Proof. By letting $\xi$ as a subdivided backbone DNA network $\operatorname{SBB}_{\mathrm{DNA}}(n)$, from Table 3 , we know

$$
\begin{aligned}
& \chi_{k}(\xi)=\sum_{g h \in E(\xi)}\left[d_{g}+d_{h}\right]^{k}, \\
& \chi_{k}(\xi)=10 n\left(4^{k}\right)+(6 n-6)\left(5^{k}\right) .
\end{aligned}
$$

By doing some calculations, we get

$$
\chi_{k}(\xi)=n\left\{10\left\{4^{k}\right\}+6\left\{5^{k}\right\}\right\}-6\left\{5^{k}\right\} .
$$


Theorem 35. For the subdivided backbone DNA network, $S B B_{D N A}(n)$, the first general Zagreb index is calculated by

$$
{ }^{k} M_{1}(\xi)=n\left\{10\left\{2^{k}\right\}+3\left\{2^{k}\right\}+2\left\{3^{k}\right\}\right\}-\left\{3\left\{2^{k}\right\}+2\left\{3^{k}\right\}\right\} .
$$

Proof. By letting $\xi$ as a subdivided backbone DNA network $\mathrm{SBB}_{\mathrm{DNA}}(n)$, from Table 3, we know

$$
\begin{aligned}
& { }^{k} M_{1}(\xi)=\sum_{g h \in E(\xi)}\left[d_{g}^{k-1}+d_{h}^{k-1}\right], \quad k>1, \\
& { }^{k} M_{1}(\xi)=10 n\left(2^{k}\right)+(6 n-6)\left(2^{k-1}+3^{k-1}\right),
\end{aligned}
$$

and by doing some calculations, we get

$$
{ }^{k} M_{1}(\xi)=n\left\{10\left\{2^{k}\right\}+3\left\{2^{k}\right\}+2\left\{3^{k}\right\}\right\}-\left\{3\left\{2^{k}\right\}+2\left\{3^{k}\right\}\right\} .
$$

Theorem 36. For the subdivided backbone DNA network, $S B B_{D N A}(n)$, the forgotten index is calculated by

$$
F(\xi)=2\{79 n-39\} \text {. }
$$

Proof. By letting $\xi$ as a subdivided backbone DNA network $\mathrm{SBB}_{\mathrm{DNA}}(n)$, from Table 3, we know

$$
\begin{aligned}
& F(\xi)=\sum_{g h \in E(\xi)}\left[d_{g}^{2}+d_{h}^{2}\right], \\
& F(\xi)=10 n(8)+(6 n-6)(13),
\end{aligned}
$$

and by doing some calculations, we get

$$
F(\xi)=2\{79 n-39\} .
$$

2.4. Results for the Subdivided Honeycomb Network. The honeycomb network is a hexagon. It can be made in different methods. The first honeycomb network is symbolized by $\mathrm{HC}_{(1)}$. The next honeycomb network is produced by attaching more hexagons to each of its edges. This newly formed honeycomb network is symbolized by $\mathrm{HC}_{(2)}$; similarly, the next honeycomb network is produced by attaching more hexagons to each of its edges. In this way, the newly formed honeycomb network is denoted by $\mathrm{HC}_{(3)}$. By repeating this process, we finally obtain a honeycomb network of $n$ dimensions and denote by $\mathrm{HC}_{(n)}$. The honeycomb network is being used in computer graphics, image processing, and cellular phone base stations; moreover, it is used in chemistry for the representation of benzenoid hydrocarbons. To get the subdivided honeycomb network shown in Figure 4, we insert a new node on each of its edges. The $n$-dimensional subdivided honeycomb network is symbolized by $\mathrm{SHC}_{n}$. A subdivided honeycomb network for $n=4$ is displayed in Figure 4 . The number of nodes and edges in the subdivided honeycomb networks are $15 n^{2}-3 n$ and $18 n^{2}-6 n$, respectively. We have obtained two different types of edges in $\mathrm{SHC}_{4}$ shown in Table 4, whereas Figure 4 shows $\mathrm{SHC}_{4}$.

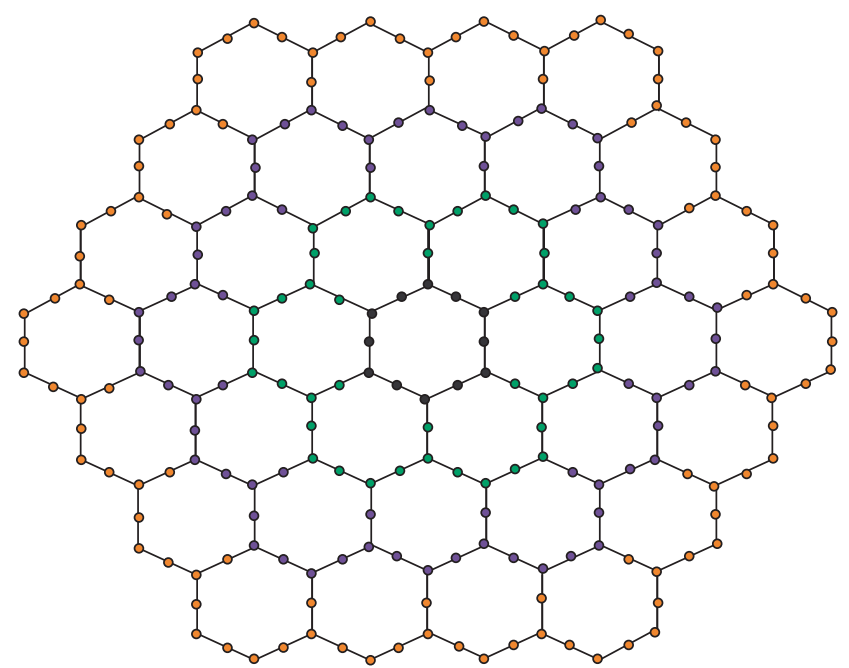

FIgURE 4: 4-dimensional $\mathrm{SHC}_{4}$.

TABLE 4: Division of edges of a graph $\xi$ found on the degree of terminating nodes of each of the edges.

\begin{tabular}{lc}
\hline$\left(d_{g}, d_{h}\right)$ for $g h \in E(\xi)$ & Number of $E(\xi)$ \\
\hline$(2,2)$ & $12 n$ \\
$(2,3)$ & $18 n(n-1)$ \\
\hline
\end{tabular}

Theorem 37. For the subdivided honeycomb network, $S H C_{n}$, the ordinary generalized geometric-arithmetic index is calculated by

$$
\mathrm{OGA}_{k}(\xi)=6\left[3 n^{2}\left\{\frac{\sqrt{24}}{5}\right\}^{k}+n\left\{2-3\left\{\frac{\sqrt{24}}{5}\right\}^{k}\right\}\right]
$$

Proof. By letting $\xi$ as a subdivided honeycomb network $\mathrm{SHC}_{n}$, from Table 4, we know

$$
\begin{aligned}
& \operatorname{OGA}_{k}(\xi)=\sum_{g h \in E(\xi)}\left[\frac{\sqrt{4 d_{g} d_{h}}}{d_{g}+d_{h}}\right]^{k}, \\
& \operatorname{OGA}_{k}(\xi)=(12 n)\left[\frac{\sqrt{16}}{2+2}\right]^{k}+18 n(n-1)\left[\frac{\sqrt{24}}{5}\right]^{k},
\end{aligned}
$$

and by doing some calculations, we get

$$
\mathrm{OGA}_{k}(\xi)=6\left[3 n^{2}\left\{\frac{\sqrt{24}}{5}\right\}^{k}+n\left\{2-3\left\{\frac{\sqrt{24}}{5}\right\}^{k}\right\}\right]
$$

Theorem 38. For the subdivided honeycomb network, $\mathrm{SHC}_{n}$, the first and second Gourava indices are calculated by $G O_{1}(\xi)=198 n^{2}-102 n$ and $G_{2}(\xi)=540 n^{2}-492 n$. 
Proof. By letting $\xi$ as a subdivided honeycomb network $\mathrm{SHC}_{n}$, from Table 4, we know

$$
\begin{aligned}
& \mathrm{GO}_{1}(\xi)=\sum_{g h \in E(\xi)}\left[\left(d_{g}+d_{h}\right)+\left(d_{g} d_{h}\right)\right], \\
& \mathrm{GO}_{2}(\xi)=\sum_{g h \in E(\xi)}\left[\left(d_{g}+d_{h}\right)+\left(d_{g} d_{h}\right)\right],
\end{aligned}
$$

and by doing some calculations, we get

$$
\begin{aligned}
\mathrm{GO}_{1}(\xi) & =(12 n)[(4)+(4)]+18 n(n-1)[(5)+(6)] \\
& =198 n^{2}-102 n, \\
\mathrm{GO}_{2}(\xi) & =(12 n)[16]+18 n(n-1)[30]=540 n^{2}-492 n .
\end{aligned}
$$

Theorem 39. For the subdivided honeycomb network, $S H C_{n}$, the first and second hyper-Gourava indices are calculated by $\mathrm{HGO}_{1}(\xi)=2178 n^{2}-1410 n$ and $\mathrm{HGO}_{2}(\xi)=16200 n^{2}-$ $13128 n$.

Proof. By letting $\xi$ as the subdivided honeycomb network $\mathrm{SHC}_{n}$, from Table 4, we know

$$
\begin{aligned}
& \operatorname{HGO}_{1}(\xi)=\sum_{g h \in E(\xi)}\left[\left(d_{g}+d_{h}\right)+\left(d_{g} d_{h}\right)\right]^{2}, \\
& \mathrm{HGO}_{2}(\xi)=\sum_{g h \in E(\xi)}\left[\left(d_{g}+d_{h}\right)+\left(d_{g} d_{h}\right)\right]^{2},
\end{aligned}
$$

and by doing some calculations, we get

$$
\begin{aligned}
\mathrm{HGO}_{1}(\xi) & =(12 n)[64]+18 n(n-1)[121] \\
& =2178 n^{2}-1410 n, \\
\mathrm{HGO}_{2}(\xi) & =(12 n)[256]+18 n(n-1)[900] \\
& =16200 n^{2}-13128 n .
\end{aligned}
$$

Theorem 40. For the subdivided honeycomb network, $S H C_{n}$, the general Randic' index is calculated by

$$
R_{\gamma}\left(S H C_{n}\right)= \begin{cases}3 n^{2}, & \text { for } \gamma=-1, \\ 3\left\{n^{2} \sqrt{6}+n\{2-\sqrt{6}\}\right\}, & \text { for } \gamma=-\frac{1}{2}, \\ 6\left\{3 n^{2} \sqrt{6}+n\{4-3 \sqrt{6}\}\right\}, & \text { for } \gamma=\frac{1}{2}, \\ 108 n^{2}-60 n, & \text { for } \gamma=1 .\end{cases}
$$

Proof. By letting $\xi$ as a subdivided honeycomb network $\mathrm{SHC}_{n}$ of $n$ dimensions, we have the order and size of $\xi$ in $\mathrm{SHC}_{n}$ as $\left|V\left(\mathrm{SHC}_{n}\right)\right|=15 n^{2}-3 n$ and $\left|E\left(\mathrm{SHC}_{n}\right)\right|=$ $18 n^{2}-6 n$, respectively. We know that

$$
R_{\gamma}(\xi)=\sum_{g h \in E(\xi)}\left(d_{g} \cdot d_{h}\right)^{\gamma},
$$

for $\gamma=\{-1,1,-1 / 2,1 / 2\}$.

Case 1: if $\gamma=-1$, the application of Randic' index $R_{\gamma}(\xi)$

$$
R_{-1}(\xi)=\sum_{g h \in E(\xi)} \frac{1}{d_{g} d_{h}},
$$

using (146). From Table 4, we obtain $R_{-1}(\xi)=$ $12 n(4)^{-1}+18 n(n-1)(6)^{-1}$.

By doing some calculations, we obtain $R_{-1}(\xi)=3 n^{2}$.

Case 2: if $\gamma=-(1 / 2)$, the application of Randic' index $R_{\gamma}(\xi)$

$$
R_{-(1 / 2)}(\xi)=\sum_{g h \in E(\xi)} \frac{1}{\sqrt{\left(d_{g} \cdot d_{h}\right)}}
$$

using (146). From Table 4, we obtain $R_{-(1 / 2)}(\xi)=$ $12 n(1 / \sqrt{4})+18 n(n-1)(1 / \sqrt{6})$.

By doing some calculations, we obtain $R_{-(1 / 2)}(\xi)=3\left\{n^{2} \sqrt{6}+n\{2-\sqrt{6}\}\right\}$.

Case 3: if $\gamma=(1 / 2)$, the application of Randic index $R_{\gamma}(\xi)$

$$
R_{(1 / 2)}(\xi)=\sum_{g h \in E(\xi)} \sqrt{d_{g} \cdot d_{h}},
$$

using (146). From Table 4, we obtain $R_{(1 / 2)}(\xi)=12 n \sqrt{4}+18 n(n-1) \sqrt{6}$.

By doing some calculations, we obtain $R_{(1 / 2)}(\xi)=6\left\{3 n^{2} \sqrt{6}+n\{4-3 \sqrt{6}\}\right\}$.

Case 4: if $\gamma=1$, the application of Randic' index $R_{\gamma}(\xi)$

$$
R_{1}(\xi)=\sum_{g h \in E(\xi)}\left(d_{g} \cdot d_{h}\right)^{1},
$$

using (146). From Table 4, we obtain $R_{1}(\xi)=$ $12 n(4)+18 n(n-1)(6)$.

By doing some calculations, we obtain $R_{1}(\xi)=108 n^{2}-60 n$.

Theorem 41. For the subdivided honeycomb network, $S H C_{n}$, the harmonic index is calculated by

$$
\mathrm{HI}(\xi)=\frac{36 n^{2}-6 n}{5} .
$$

Proof. By letting $\xi$ as a subdivided honeycomb network $\mathrm{SHC}_{n}$, from Table 4, we know

$$
\mathrm{HI}(\xi)=\sum_{g h \in E(\xi)} \frac{2}{d_{g}+d_{h}},
$$

and by doing some calculations, we get 


$$
\mathrm{HI}(\xi)=(12 n)\left[\frac{1}{2}\right]+18 n(n-1)\left[\frac{2}{5}\right]=\frac{36 n^{2}-6 n}{5}
$$

Theorem 42. For the subdivided honeycomb network, $\mathrm{SHC}_{n}$, the general version of the harmonic index is calculated by

$$
H_{k} I(\xi)=6\left[3 n^{2}\left\{\frac{2}{5}\right\}^{k}+n\left\{\frac{2}{2^{k}}-3\left\{\frac{2}{5}\right\}^{k}\right\}\right] \text {. }
$$

Proof. By letting $\xi$ as a subdivided honeycomb network $\mathrm{SHC}_{n}$, from Table 4, we know

$$
H_{k} I(\xi)=\sum_{g h \in E(\xi)}\left[\frac{2}{d_{g}+d_{h}}\right]^{k}
$$

and by doing some calculations, we get

$$
\begin{aligned}
H_{k} I(\xi) & =(12 n)\left[\frac{2}{2+2}\right]^{k}+18 n(n-1)\left[\frac{2}{2+3}\right]^{k}, \\
& =6\left[3 n^{2}\left\{\frac{2}{5}\right\}^{k}+n\left\{\frac{2}{2^{k}}-3\left\{\frac{2}{5}\right\}^{k}\right\}\right] .
\end{aligned}
$$

Theorem 43. For the subdivided honeycomb network, $S H C_{n}$, the atom-bond connectivity index is calculated by

$$
A B C(\xi)=n \sqrt{2}[9 n-3]
$$

Proof. By letting $\xi$ as a subdivided honeycomb network $\mathrm{SHC}_{n}$, from Table 4, we know

$$
A B C(\xi)=\sum_{g h \in E(\xi)} \sqrt{\frac{d_{g}+d_{h}-2}{d_{g} d_{h}}},
$$

and by doing some calculations, we get

$$
\begin{aligned}
A B C(\xi) & =12 n \sqrt{\frac{2+2-2}{4}}+18 n(n-1) \sqrt{\frac{2+3-2}{6}}, \\
& =n \sqrt{2}[9 n-3] .
\end{aligned}
$$

Theorem 44. For the subdivided honeycomb network, $S H C_{n}, S K, S K_{1}$, and $S K_{2}$ indices are calculated by $S K(\xi)=$ $45 n^{2}-21 n, \quad S K_{1}(\xi)=54 n^{2}-30 n$, and $S K_{2}(\xi)=(1 / 2)$ $\left(225 n^{2}-129 n\right)$, respectively.

Proof. By letting $\xi$ as a subdivided honeycomb network $\mathrm{SHC}_{n}$, from Table 4, we know

$$
\begin{aligned}
& S K(\xi)=\sum_{g h \in E(\xi)}\left[\frac{d_{g}+d_{h}}{2}\right], \\
& S K_{1}(\xi)=\sum_{g h \in E(\xi)}\left[\frac{d_{g} d_{h}}{2}\right], \\
& S K_{2}(\xi)=\sum_{g h \in E(\xi)}\left[\frac{d_{g}+d_{h}}{2}\right]^{2},
\end{aligned}
$$

and by doing some calculations, we get

$$
\begin{aligned}
& S K(\xi)=(12 n)(2)+18 n(n-1)\left(\frac{5}{2}\right)=45 n^{2}-21 n, \\
& S K_{1}(\xi)=(12 n)(2)+18 n(n-1)(3)=54 n^{2}-30 n, \\
& S K_{2}(\xi)=(12 n)(4)+18 n(n-1)\left(\frac{25}{4}\right)=\frac{1}{2}\left(225 n^{2}-129 n\right) .
\end{aligned}
$$

Theorem 45. For the subdivided honeycomb network, $S H C_{n}$, the sum-connectivity index is calculated by

$$
\chi_{-(1 / 2)}(\xi)=\frac{1}{\sqrt{5}}\left[18 n^{2}+n\{6 \sqrt{5}-18\}\right] .
$$

Proof. By letting $\xi$ as a subdivided honeycomb network $\mathrm{SHC}_{n}$, from Table 4, we know

$$
\begin{aligned}
& \chi_{-(1 / 2)}(\xi)=\sum_{g h \in E(\xi)}\left[d_{g}+d_{h}\right]^{-(1 / 2)}, \\
& \chi_{-(1 / 2)}(\xi)=(12 n)\left(\frac{1}{2}\right)+18 n(n-1)\left(\frac{1}{\sqrt{5}}\right),
\end{aligned}
$$

and by doing some calculations, we get

$$
\chi_{-(1 / 2)}(\xi)=\frac{1}{\sqrt{5}}\left[18 n^{2}+n\{6 \sqrt{5}-18\}\right] .
$$

Theorem 46. For the subdivided honeycomb network, $\mathrm{SHC}_{n}$, the general sum-connectivity index is calculated by

$$
\chi_{k}(\xi)=18\left\{5^{k}\right\} n^{2}+6 n\left\{2\left\{4^{k}\right\}-3\left\{5^{k}\right\}\right\} .
$$

Proof. By letting $\xi$ as a subdivided honeycomb network $\mathrm{SHC}_{n}$, from Table 4, we know

$$
\begin{aligned}
& \chi_{k}(\xi)=\sum_{g h \in E(\xi)}\left[d_{g}+d_{h}\right]^{k}, \\
& \chi_{k}(\xi)=(12 n)\left(4^{k}\right)+18 n(n-1)\left(5^{k}\right),
\end{aligned}
$$

and by doing some calculations, we get 


$$
\chi_{k}(\xi)=18\left\{5^{k}\right\} n^{2}+6 n\left\{2\left\{4^{k}\right\}-3\left\{5^{k}\right\}\right\} .
$$

Theorem 47. For the subdivided honeycomb network, $S H C_{n}$, the first general Zagreb index is calculated by

${ }^{k} M_{1}(\xi)=18 n^{2}\left\{2^{k-1}+3^{k-1}\right\}+6 n\left\{2\left\{2^{k}\right\}-3 n\left\{2^{k-1}+3^{k-1}\right\}\right\}$.

Proof. By letting $\xi$ as a subdivided honeycomb network $\mathrm{SHC}_{n}$, from Table 4, we know

$$
\begin{aligned}
& { }^{k} M_{1}(\xi)=\sum_{g h \in E(\xi)}\left[d_{g}^{k-1}+d_{h}^{k-1}\right], \quad k>1, \\
& { }^{k} M_{1}(\xi)=(12 n)\left(2^{k}\right)+18 n(n-1)\left(2^{k-1}+3^{k-1}\right),
\end{aligned}
$$

and by doing some calculations, we get

$$
{ }^{k} M_{1}(\xi)=18 n^{2}\left\{2^{k-1}+3^{k-1}\right\}+6 n\left\{2\left\{2^{k}\right\}-3 n\left\{2^{k-1}+3^{k-1}\right\}\right\} .
$$

Theorem 48. For the subdivided honeycomb network, $S H C_{n}$, the forgotten index is calculated by

$$
F(\xi)=2 n\{117 n-69\} .
$$

Proof. By letting $\xi$ as a subdivided honeycomb network $\mathrm{SHC}_{n}$, from Table 4, we know

$$
\begin{aligned}
& F(\xi)=\sum_{g h \in E(\xi)}\left[d_{g}^{2}+d_{h}^{2}\right], \\
& F(\xi)=(12 n)(8)+18 n(n-1)(13),
\end{aligned}
$$

and by doing some calculations, we get $F(\xi)=$ $2 n\{117 n-69\}$.

\section{Conclusions}

In this paper, we have computed the topological indices (degree based) such as ordinary generalized geometricarithmetic (OGA) index, first and second Gourava indices, first and second hyper-Gourava indices, general Randic' index $R_{\gamma}(\xi)$, for $\gamma=\{ \pm 1, \pm(1 / 2)\}$, harmonic index, general version of the harmonic index, atom-bond connectivity (ABC) index, $\mathrm{SK}, \mathrm{SK}_{1}$, and $\mathrm{SK}_{2}$ indices, sum-connectivity index, general sum-connectivity index, and first general Zagreb and forgotten topological indices for different kinds of chemical networks such as the subdivided polythiophene network, subdivided hexagonal network, subdivided backbone DNA network, and subdivided honeycomb network. The above computed topological indices are used as molecular descriptors in the construction of "quantitative structure-activity relationships and quantitative structureproperty relationships." These indices give us results that can be correlated with the molecular structures to understand their chemical and physical properties.

For the next research papers, our goal is to compute more topological indices for some new graphs to know their topologies.

\section{Data Availability}

No data were used to support this study.

\section{Disclosure}

This paper has not been published elsewhere, and it will not be submitted anywhere else for publication.

\section{Conflicts of Interest}

The authors declare that they have no conflicts of interest.

\section{Authors' Contributions}

All authors contributed equally to this work.

\section{Acknowledgments}

The authors like to show their gratitude to the concerned people for sharing their pearls of wisdom with them during this research work.

\section{References}

[1] A. Ali, L. Zhong, and I. Gutman, "Harmonic index and its generalizations: extremal results and bounds," MATCH Communications in Mathematical and in Computer, vol. 81, pp. 249-311, 2019.

[2] A. Ali, I. Gutman, E. Milovanovic, and I. Milovanovic, "Sum of powers of the degrees of graphs: extremal results and bounds," MATCH Communications in Mathematical and in Computer, vol. 80, pp. 5-84, 2018.

[3] A. Ali, K. C. Das, D. Dimitrov, and B. Furtula, "Atom-bond connectivity index of graphs: a review over extremal results and bounds," Discrete Mathematics Letters, vol. 5, pp. 68-93, 2021.

[4] Z. S. Mufti, M. F. Nadeem, W. Gao, and Z. Ahmad, "Topological study of the para-line graphs of certain pentacene via topological indices," Open Chemistry, vol. 16, no. 1, pp. 1200-1206, 2018.

[5] B. Borovicanin, K. C. Das, B. Furtula, and I. Gutman, "Bounds for zagreb indices," MATCH Communications in Mathematical and in Computer, vol. 78, pp. 17-100, 2017.

[6] A. Portilla, J. M. Rodriguez, and J. M. Sigarreta, "Recent lower bounds for geometric-arithmetic index," Discrete Mathematics Letters, vol. 1, pp. 59-82, 2019.

[7] B. Zhou, I. Gutman, B. Furtula, and Z. Du, "On two types of geometric-arithmetic index," Chemical Physics Letters, vol. 482, no. 1-3, pp. 153-155, 2009.

[8] D. Vukičević and B. Furtula, "Topological index based on the ratios of geometrical and arithmetical means of end-vertex degrees of edges," Journal of Mathematical Chemistry, vol. 46, no. 4, pp. 1369-1376, 2007. 
[9] G. Fath-Tabar, B. Furtula, and I. Gutman, "A new geometricarithmetic index," Journal of Mathematical Chemistry, vol. 47, no. 1, pp. 477-486, 2010.

[10] K. C. Das, I. Gutman, and B. Furtula, "Survey on geometric-arithmetic indices of graphs," MATCH Communications in Mathematical and in Computer Chemistry, vol. 65, pp. 595-644, 2011.

[11] Y. Yuan, B. Zhou, and N. Trinajstić, "On geometric-arithmetic index," Journal of Mathematical Chemistry, vol. 47, no. 2, pp. 833-841, 2010.

[12] M. Eliasi and I. Ali, "On ordinary generalized geometric- arithmetic index," Applied Mathematics Letters, vol. 24, no. 4 , pp. 582-587, 2011.

[13] V. R. Kulli, "The Gourava indices and coindices of Graphs," Annals of Pure and Applied Mathematics, vol. 14, no. 1, pp. 33-38, 2017.

[14] V. R. Kulli, "On hyper-Gourava indices and coindices," International Journal of Mathematical Archive, vol. 8, no. 12, pp. 116-120, 2017.

[15] M. Randi'c, "On characterization of molecular branching," Journal of the American Chemical Society, vol. 97, pp. 66096615, 1975.

[16] M. R. Farahani, "On the randic and sum-connectivity index of nanotubes," Annals of West University of Timisoara Mathematics, vol. 51, no. 2, pp. 29-37, 2013.

[17] X. Li and Y. Shi, "A survey on the randic index," MATCH Communications in Mathematical and in Computer Chemistry, vol. 59, pp. 127-156, 2008.

[18] B. Bollobás and P. Erdos, "Graphs of extremal weights," Ars Combinatoria, vol. 50, pp. 225-233, 1998.

[19] B. Wu and L. Zhang, "Unicyclic graphs with minimum general Randi'c index," MATCH Communications in Mathematical and in Computer Chemistry, vol. 54, pp. 455464, 2005.

[20] X. Li and I. Gutman, Mathematical Aspects of Randi'c-type Molecular Structure Descriptors, University of Kragujevac, Kragujevac, Serbia, 2006.

[21] Y. Hu, X. Li, and Y. Yuan, "Trees with minimum general Randi'c index," MATCH Communications in Mathematical and in Computer Chemistry, vol. 52, pp. 119-128, 2004.

[22] L. Zhong, "The harmonic index for graphs," Applied Mathematics Letters, vol. 25, no. 3, pp. 561-566, 2012.

[23] S. Fajtlowicz, "On conjectures of Graffti - II," Congressus Numerantium, vol. 60, pp. 187-197, 1987.

[24] L. Yan, W. Gao, and J. Li, "General harmonic index and general sum connectivity index of polyomino chains and nanotubes," Journal of Computational and Theoretical Nanoscience, vol. 12, no. 10, pp. 3940-3944, 2015.

[25] E. Estrada, L. Torres, L. Rodriguez, and I. Gutman, “An atombond connectivity index: modelling the enthalpy of formation of alkanes," Indian Journal of Chemistry, vol. 37, pp. 849-855, 1998.

[26] E. Estrada, "Atom-bond connectivity and the energetic of branched alkanes," Chemical Physics Letters, vol. 463, no. 4-6, pp. 422-425, 2008.

[27] V. S. Shegehalli and R. Kanabur, "Computation of new degree-based topological indices of graphene," Journal of Mathematics, vol. 5, p. 6, Article ID 4341919, 2016.

[28] B. Lučić, N. Trinajstić, and B. Zhou, "Comparison between the sum-connectivity index and product-connectivity index for benzenoid hydrocarbons," Chemical Physics Letters, vol. 475, no. 1-3, pp. 146-148, 2009.
[29] B. Zhou and N. Trinajstić, "On general sum-connectivity index," Journal of Mathematical Chemistry, vol. 47, no. 1, pp. 210-218, 2010.

[30] H. M. Awais, M. Javaid, and A. Ali, "Frist general zagreb index of generalized f-sum graphs," Discrete Dynamics in Nature and Society, vol. 2020, Article ID 2954975, 16 pages, 2020.

[31] J.-B. Liu, S. Javed, M. Javaid, and K. Shabbir, "Computing first general Zagreb index of operations on graphs," IEEE Access, vol. 7, pp. 47494-47502, 2019.

[32] S. M. Hosamani and I. Gutman, "Zagreb indices of transformation graphs and total transformation graphs," Applied Mathematics and Computation, vol. 247, pp. 1156-1160, 2014.

[33] S. Zhang and H. Zhang, "Unicyclic graphs with the first three smallest and largest first general zagreb index," MATCH Communications in Mathematical and in Computer Chemistry, vol. 55, pp. 427-438, 2006.

[34] X. Li and J. Zheng, "A unified approach to the extremal trees for different indices," MATCH Communications in Mathematical and in Computer Chemistry, vol. 54, pp. 195-208, 2005.

[35] X. Li and H. Zhao, "Trees with the first three smallest and largest generalized topological indices," MATCH Communications in Mathematical and in Computer Chemistry, vol. 50, pp. 57-62, 2004.

[36] B. Furtula and I. Gutman, "A forgotten topological index," Journal of Mathematical Chemistry, vol. 53, no. 4, pp. 11841190, 2012.

[37] H. Abdo, D. Dimitrov, and I. Gutman, "On extremal trees with respect to the F-index," 2013, https://arxiv.org/abs/1509. 03574 .

[38] N. De, A. Nayeem, and A. Pal, "F-index of some graph operations," 2015, https://arxiv.org/abs/1511.06661.

[39] Y. Kongyang, C. Xiaosong, J. Zhepeng, Z. Cong, W. Dacheng, and L. Yunqi, "Two-dimensional cross-linked polythiophene network," Journal of Materials Chemistry C, vol. 7, 2019.

[40] A. Ghosh and M. Bansal, "A glossary of DNA structures from A to Z," Acta Crystallographica Section D, vol. 59, no. 4, pp. 620-626, 2003. 